\title{
LA EDUCACIÓN AMBIENTAL A TRAVÉS DEL TURISMO ECOLÓGICO EN ADULTOS MAYORES EN LA LOCALIDAD DE FOMENTO, VILLA CLARA, CUBA CON EL USO DE MATERIAL AUDIOVISUAL
}

THE ENVIRONMENTAL EDUCATION THROUGH THE ECOLOGICAL TOURISM IN GREATER ADULTS IN THE LOCALITY OF FOMENTO, VILLA CLARA, CUBA WITH THE USE OF AUDIOVISUAL MATERIAL

\author{
Natacha Coca Bernal \\ nlcoca@nauta.cu \\ Carinebys Alpizar Ortueta \\ Marcial Dayan Alonso García
}


Para citar este artículo:

Coca Bernal , N., García Iglesias, M. de la C., Alpizar Ortueta, C., Alonso García, M. D., Gody Morffi , D., \& Hernández Heredia, D. de la C. (2020). La educación ambiental a través del turismo ecológico en adultos mayores en la localidad de Fomento, Villa Clara, Cuba con el uso de material audiovisual. ESPACIO I+D, INNOVACIÓN MÁS DESARROLLO, 9(24). https://doi.org/10.31644/IMASD.24.2020.a01

\section{RESUMEN}

La investigación se aplicó en el municipio de Fomento, Villa Clara, Cuba durante un año con el objetivo de: Construir, a través de una investigaciónacción-participativa, una Estrategia Educativa de turismo ecológico, con el uso de material audiovisual en el adulto mayor para estilos de vida sanos y saludables. Se utilizaron técnicas de investigación-acción-participativa: la observación participante, triangulación de fuentes, diario del investigador, grupos focales de discusión y estudio de caso. Como resultados de la Estrategia Educativa con el turismo ecológico, se redujo en un porcentaje considerable los niveles de estrés generados en los adultos mayores, las enfermedades crónicas se mantuvieron estables. Además, cambió su percepción sobre el turismo, que con el uso de la Educación Ambiental puede adaptarse a las discapacidades y potencialidades de estas edades. También reconocieron la importancia de la ingesta de frutas y vegetales. Esta experiencia permitió la socialización de los adultos mayores y un acercamiento a sus familiares, y en los grupos focales de discusión, se evidenció cómo se manifestaba su vida sexual pasada presente y futura. Con todas sus vivencias se construyeron materiales audiovisuales.

\section{Palabras clave}

Turismo ecológico, educación ambiental, adulto mayor y material audiovisual. 
The investigation was applied in the municipality of Promotion, Villa Clara, Cuba during a year with the objective of: To construct through an investigation - action - participation an Educative Strategy of ecological tourism with the use of audio-visual material in the greater adult for healthy and healthful styles of life.Investigation-action-paticipacio'n techniques were used:the participant observation, triangulation of sources, newspaper of the investigator, focal groups of discussion and study of case.Like results of the Educative Strategy with the ecological tourism, it was reduced in a considerable percent the levels of stress generated in the greater adults, the chronic diseases stayed stable. In addition, it changed its perception on the tourism, that with the use of the Environmental Education can adapt to the few capacities and potentialities of these ages. Also they recognized the importance of the ingestion of fruits and vegetables. This experience allowed to the socialization of the greater adults and an approach to its relatives, and in the focal groups of discussion, it was demonstrated how its sexual life was pronounced last future present and. With all their experiences audio-visual materials were constructed.

\section{Keywords}

Ecological tourism, environmental, adult education greater and material audio-visual. 
L os adultos mayores son un grupo etario que oscila de los 60 años en adelante. "Cuba, con una población con edad de 60 o más años por encima del $15 \%$, es un país donde toda persona que alcanza los 60 años de edad, y los supera, es considerada un adulto mayor" (GalbánI, Soberats, \& Díaz-Canel, 2006). Gracias a los avances de la ciencia y la tecnología, las personas viven más años, pero no con la calidad de vida que se desea.

Los adultos mayore,s en su gran mayoría, no se adaptan al proceso de envejecimiento, las capacidades físicas y mentales tienden a disminuir, pero, de acuerdo con su condición física y mental, algunas personas son capaces de realizar múltiples actividades. La depresión, la falta de ejercicios físicos y el suicidio son frecuentes en esta etapa de la vida. "La depresión en el adulto mayor tiene un efecto sistémico y los ancianos deprimidos parecen tener mayor susceptibilidad para las enfermedades, puesto que esta patología ocasiona cambios neurológicos, hormonales e inmunológicos...en las emociones se acompaña de complejas modificaciones neuroendocrinas con secuencia de eventos fisiopatológicos” (Aguilar-Navarroa \& Ávila-Funesa, 2007).

Los datos indican que Cuba es un país envejecido, razón por la cual el sistema nacional de salud se ha preparado para las enfermedades más frecuentes en estas edades. Desde el punto de vista psicológico es difícil de llegar a cada persona, aun cuando se les brinda alimentación, consultas y medicamentos gratuitos a aquellos de bajos ingresos, la esfera afectiva motivacional no se atiende de la misma forma. "El abordaje del envejecimiento supone implementar políticas y programas públicos para la atención integral de la persona adulta mayor. Cuba tiene como desafío mejorar el bienestar y la calidad de vida de este grupo, por ello se incluye el envejecimiento poblacional como un eje temático sobre el cual todos los órganos e instituciones cubanas están abocados a trabajar" (Mercedes, Sánchez, Somonte, \& Cabrera, 2014).

Este grupo de personas renuncia por voluntad propia a las reuniones sociales, se aíslan y su vida va perdiendo el sentido. Fueron excelentes trabajadores, esposos, hijos, pero ya estas tareas no las desempeñan, y tampoco ocupan su tiempo de forma sana y didáctica. Por otra parte, las personas que los rodean las menosprecian, buscan apoyo y asesoría en personas más jóvenes, aun cuando éstos sean menos capacitados que los adultos mayores. "La longevidad creciente tiene exigencias propias que no pueden desconocerse y se debe asegurar que el alto costo necesario para llegar a viejo se vea compensado con una vida con calidad (Vera, 2007).

En la localidad de Fomento, provincia de Villa Clara, se pudo constatar a través de diferentes métodos y técnicas que:

- $\quad$ Este grupo presenta bajo nivel adquisitivo, debido a falta de opciones laborales acordes con su edad. 
- Presentan enfermedades crónicas como: diabetes, hipertensión, cardiopatías y trastornos emocionales, entre otras.

- Estrés postraumáticos por la viudez.

- $\quad$ Riesgos por accidentes y riesgos de suicidio.

- No realizan una distracción sana debido a que la cultura y recreación está encaminada principalmente al grupo de entre 15 a 40 años. La música y bailes no son apropiados a sus gustos y motivaciones.

- Esta población presenta un alto nivel cultural pero están desactualizados en sus ramas de estudio.

- Los diseñadores de modas trabajan en su gran mayoría para personas jóvenes y no tienen en cuenta las edades avanzadas; el diseño de ropa y calzado no responde a sus gustos y a las necesidades que se derivan de las enfermedades propias de esta edad.

- En cuanto a la sexualidad, no se comunican con libertad y sin perjuicio de su sexualidad, han perdido su pareja, es difícil buscar una nueva relación y expresar su amor y empatía por el sexo contrario.

- Presentan desmotivación, apatía, sentimiento de desamparo con respecto a sus familiares y a la población en general.

- No realizan ejercicios físicos de acuerdo con su edad, por lo que presentan problemas con el peso, obesidad o bajo peso.

- No utilizan de forma sana el tiempo libre.

- Desconocen las potencialidades turísticas de su localidad.

Estas insipiencias y conductas fueron tomadas del grupo poblacional de adultos mayores, de la localidad de Fomento, provincia de Villa Clara; lo cual incitó a investigar la construcción de una Estrategia Educativa, desde una investigación-acción-participativa. Estas necesidades fueron detectadas con los siguientes métodos:

\section{MÉTODOS}

En la investigación se utilizaron técnicas de investigación-acción-participativa descrita en la estrategia investigativa de Kemmis (1988) que, "se basa en la noción de una espiral de autorreflexión de ciclos de planeamiento, acción, observación y reflexión. Expresa un compromiso al perfeccionamiento de las prácticas y es colaborativa".

Se aplicó el análisis de documentos dirigido a valorar aspectos del contenido turístico ecológico, así como la bibliografía existente. La observación participante, durante toda la investigación en las etapas de: diagnóstico, planificación, ejecución, control y evaluación de la Estrategia Educativa. Se utilizó para recopilar datos en el escenario, con los adultos mayores, familia y especialistas. 
La triangulación de fuentes para determinar los contenidos de cada uno de los subsistemas a partir de la bibliografía, resultados de estudios de esta ciencia, registros históricos del estudio del turismo ecológico y de los adultos mayores.

El diario del investigador, se utilizó para recopilar datos cualitativos detallados en cada una de las etapas de la Estrategia Educativa, facilitando su posterior análisis. Registro de forma ordenada, cronológicamente, permitiendo hipervínculos a los documentos, videos y fotos recopilados en 5 DVD de evidencia gráfica.

Los grupos infórmales de discusión, para recolectar datos cualitativos de la construcción de la Estrategia Educativa. Capacitar metodológicamente a los adultos mayores en cuanto a los contenidos del turismo ecológico. Vincularlos afectivamente con los objetivos, misiones y acciones propuestos, tomando en cuenta sus experiencias; para determinar las debilidades, fortalezas, dar sugerencias. Permitió realizar anécdotas de su vida pasada, sus añoranzas y frustraciones, así como crear planes de acciones para el turismo ecológico.

El estudio de caso para evaluar la Estrategia Educativa, permite evidenciar los conocimientos adquiridos, la esfera afectiva motivacional, la salud física y mental. Diferentes autores hacen referencia a los resultados científicos en la investigación: Castellanos, (1998); R. A. González, Y1l, \& Curiel, (2003); Kemmis, (1988); Ruiz (1999), (2000), (2009); Sampier, (2006); Silva, (2007); Valle, (2007); Rodríguez, Gil, \& García, (1996) sin embargo, para Llanes (2012) dice que "La Estrategia Educativa es un conjunto de procedimientos de investigación aplicable a cualquier ciencia, es la manera de alcanzar un objetivo con determinado procedimiento para ordenar la actividad, permite reproducir de manera consciente el objeto estudiado. La Estrategia Educativa se encuentra indisolublemente unido a la teoría."

La Estrategia Educativa, como resultado científico que emanó de la práctica pedagógica, está en concordancia con los sujetos estudiados, por lo cual, se define el siguiente objetivo general: Construir a través de una investigación- acción -participativa una Estrategia Educativa de turismo ecológico para el adulto mayor para estimular estilos de vida sanos y saludables.

La población oscila de los 60 a 75 años de edad: 35 jubilados del municipio de Fomento; se realizó la selección de forma intencional y no probabilística debido a que la Estrategia Educativa responde a las particularidades de la personalidad de estas edades, sus gustos y motivaciones. 


\section{DESARROLLO}

Fomento se encuentra en la provincia de Sancti Spíritus, limitando al Norte con el municipio de Placetas, al Este con Cabaiguán, al sur con Sancti Spíritus y Trinidad y al Oeste con Manicaragua en la provincia de Villa Clara, en Cuba. Lo atraviesan los ríos de Agabama, Mabujina, Cangrejo y varios arroyos. En la actualidad cuenta con varios poblados dentro de los que se encuentran: El Pedrero, Agabama, La Guanábana, La Hormiga y Gavilanes. La principal actividad de la región era la industria azucarera y el cultivo de caña de azúcar, pero con las modificaciones en esta industria se hizo necesario expandirse hacia otras.

La localidad cuenta con un total de 335765 habitantes (Cuba, 2015), de ellos 17123 son varones y 16453 mujeres. Según datos de este Censo de Población y Viviendas (2015), el 18,3\% de la población de Cuba tiene 60 años y más, y los territorios con mayor grado de envejecimiento son Villa Clara, con el 21,9\%; La Habana, 19,9\% y Sancti Spíritus, 19,7\%. (Cuba, 2015)

Las enfermedades más recurrentes (ver tabla 1) de los adultos mayores del municipio de Fomento, se registran de modo similar a la media del país: el asma, la hipertensión y la insuficiencia renal; con alta tasa de morbilidad y discapacidad. El turismo ecológico brinda una solución posible a la compensación de las limitaciones de estas enfermedades, así como la esfera afectiva motivacional de los adultos mayores (Fomento, 2017). 
Tabla 1

Enfermedades más recurrentes del municipio de Fomento

\begin{tabular}{cccccc}
\hline Enfermedades & \multicolumn{2}{c}{ De 60-64 años } & \multicolumn{2}{c}{65 años y más } & Total \\
& Masculino & Femenino & Masculino & Femenino & \\
Diabetes mellitus tipo I & 17 & 16 & 16 & 25 & 74 \\
Diabetes Mellitus tipo II & 5 & 5 & 16 & 26 & 52 \\
Hipertensión arterial & 109 & 256 & 687 & 1352 & 2404 \\
Asma & 625 & 952 & 3096 & 4606 & 9279 \\
Insuficiencia renal crónica & 116 & 217 & 456 & 746 & 1535 \\
Hipercolesterolemia & 71 & 150 & 237 & 527 & 985 \\
Enfermedad cerebrovascular & 16 & 9 & 113 & 101 & 239 \\
Discapacitado físico motor & 109 & 256 & 687 & 1352 & 2404 \\
Discapacitados auditivos & 625 & 952 & 3096 & 4606 & 9279 \\
Cáncer & 116 & 217 & 456 & 746 & 1535 \\
Cáncer de próstata & 71 & 150 & 237 & 527 & 985 \\
Cáncer de colon & 36 & 21 & 209 & 236 & 502 \\
Cáncer de mama & 2 & 15 & 3 & 103 & 123 \\
Obesidad & 32 & 50 & 193 & 271 & 546 \\
Sedentarismo & 48 & 53 & 106 & 250 & 457 \\
Riesgo por consumo de alcohol & 45 & 69 & 152 & 345 & 611 \\
Tabaquismo & 153 & 17 & 339 & 23 & 532 \\
\hline
\end{tabular}

Fuente: Fomento (2017) Informe de Dispensarización de la población por zona y consultorio médico. Material no publicado.

Asumimos que las fases y etapas declaradas por Rodríguez, G., Gil, J., \& García, E. (1996); fase preparatoria, trabajo de campo, analítica e informativa, sonlas más propicias para abordar el estudio del objeto, dada su naturaleza, las características y particularidades de los sujetos que han servido para desarrollar la investigación, la conformación de los resultados principales, los antecedentes en estudios de este tipo y las posibilidades de los métodos empíricos empleados.

La muestra estudiada arrojó los siguientes resultados: 10 cardiópatas, 12 hipertensos, 7 diabéticos, 2 con limitaciones físicas motores, 1 hipoacusia, 3 trastornos circulatorios, 1 trastorno psiquiátrico, 5 obesos, 3 bajo peso. Se pudo constatar que 7 adultos mayores padecían más de una enfermedad; a su vez existen 3 de ellos sin ningún padecimiento.

Para realizar esta investigación se aplicó el enfoque cualitativo, este posee etapas que se dieron en la práctica sin una frontera temporal definida. La preparación de la investigación no culminó con la Construcción de la Estrategia Educativa de turismo ecológico para los adultos mayores; así como tampoco la elaboración de instrumentos a partir de las necesidades surgidas de la práctica y del diagnóstico (acceso al campo), sino que se enriqueció 
durante la propia observación y ejecución del turismo ecológico planificados. Como plantea Taylor (1987) "la mejor forma que tiene el investigador para sumar a los informantes a su investigación es colaborarle, escuchar sus sugerencias y vivencias".

Observación participante: Permitió determinar como potencialidades en los adultos mayores:

- Un nivel cultural alto.

- La capacitación que poseen en cuanto a la Educación patrimonial del municipio.

Como necesidades:

- Presentan alto nivel de estrés por la incertidumbre y el temor por la cercanía de la muerte.

- Se encuentran tristes y desmotivados ante las actividades cotidianas.

- Presentan un grupo de enfermedades crónicas que dificultan su movilidad.

- No realizan ejercicios físicos de forma sistemática.

- Desconocimiento de la Educación ambiental y patrimonial.

- Alimentación con alto consumo de calorías o ingesta insuficiente de alimentos sanos.

- Baja percepción de riesgo.

- Alejamiento de sus familiares y vecinos.

- No expresan su sexualidad de una forma sana.

\section{ETAPA DE DISEÑO}

En la declaración de los derechos humanos se plantea que el hombre tiene derecho a la vida, a la libertad y a la seguridad. El sistema de salud se encarga de los adultos mayores, así como de su bienestar social, cuando éstos tienen bajos ingresos. El adulto mayor tiene el derecho al reconocimiento ante su colectivo, familia y la sociedad donde vive. En estas edades se siente un desamparo ante las capacidades disminuidas. Tienen derecho a ser escuchados por su propia voz o por voceros que los representen. No puede existir ningún tipo de injerencias arbitrarias en su vida privada. La familia tiende a percibir que la vida sexual del anciano no es relevante, al culminar la vida reproductiva de los hombres y mujeres, los que le rodean menosprecian su sexualidad. Los adultos mayores tienen derecho a circular libremente y a elegir su residencia en el territorio. Cuando culmina su actividad laboral remunerada, pierden o dejan de adquirir propiedades, las familias los menosprecian por su bajo poder monetario, o no lo tienen en cuenta para la toma de decisiones, pero toda persona tiene derecho a la 
propiedad, individual y colectivamente. Las reuniones culturales tienden a contemplar a los jóvenes, los adultos mayores no son excluidos, pero no asisten porque no se contemplan sus gustos culturales.

Las excursiones se realizaron con una frecuencia mensual a los sitios de turismo ecológico del municipio, un total de cuatro excursiones en todo un año. Eran largas caminatas dosificadas en cortos tramos recorridos en una marcha lenta, para estimular el sistema circulatorio de los adultos mayores. Se hacían brindis con plantas medicinales, aromáticas y se degustaban frutas, lo que repercutió en la alimentación sana del grupo. Se tuvo la oportunidad de crear una retroalimentación con enfoque en el turismo ecológico, con un ambiente sano, en contacto con la naturaleza, lo que les permitió disminuir los niveles de estrés.

Se tuvo en cuenta que la Educación Ambiental de los adultos mayores "forma un individuo; partiendo del conocimiento, de los que se requieren valores e intereses, lo que se puede (capacidades) y lo que se debe hacer (responsabilidades), tomando como referencia su problemática particular del entorno, resultado de las relaciones que se establecen entre las dinámicas propias de los componentes de la sociedad y de la naturaleza" (Ricardo, 2006)

Los actos culturales, con el uso de música campesina: la música de los campos cubanos tiene una larga tradición que se cultiva en su gran mayoría por adultos mayores, son campesinos que tienen aptitudes musicales, tocan instrumentos, cantan y bailan. Está demostrado que realizar movimientos rítmicos sincronizados con la música estimula la motórica gruesa, la motórica fina, la kinestésica. La cultura e idiosincrasia de la localidad de Fomento tiene y un énfasis en el desarrollo local (Pérez, A., 2010). La música tradicional cubana es un sonido suave y repetitivo, que el bailador acompaña con movimientos cadenciosos de sus caderas, se puede bailar en pareja de una forma muy elegante o en solitario; los bailadores aprovechan la ocasión para vestirse con trajes de gala, utilizar guayaberas, prenda tradicional cubana. Las mujeres usan abanicos; todo esto crea un ambiente de galantería y seducción que le hace recordar a los adultos mayores sus años jóvenes. La décima cubana incluye una poesía improvisada por los cantores; la creación de la décima estimula el vocabulario activo y pasivo de los adultos mayores, estimula la memoria, estimulan el oído fonemático y los músculos que intervienen en la articulación del sonido.

En los actos culturales participaron las familisa de los adultos mayores, estudiantes de escuelas especiales; lo que permitió un acercamiento mejor o la dinámica de las relaciones interpersonales. "El hombre aprende a través de los sentidos, ya que es la puerta de entrada de toda la información” (Nico, 2016)

Cuentos inéditos de los adultos mayores fueron filmados en los intercambios donde narraron sus historias de vida. Estas historias no tienen fiabilidad, fueron fabuladas y reconstruidas. Los cuentos fueron narrados 
nuevamente en intercambios. Se crearon videos sencillos, con el uso de material audiovisual. "La creación y narración son actividades que le permite... al individuo exponer experiencias sociales... facilita la motivación de los estímulos externos” Coca, N., Martínez, P., \& Álvarez, M. (2016).

\section{Material audiovisual para la educación ambiental y el turismo ecológico}

En la realización y utilización de materiales audiovisuales, se tuvo en cuenta la cultura e identidad de la localidad, los animales y plantas autóctonos, debido a que "Las imágenes resultan motivadoras, sensibilizan y estimulan el interés de las personas hacia un tema determinado. Facilitan la instrucción, complementando las explicaciones verbales con contenidos icónicos concretos de fácil comprensión, que contribuyen a la fijación de los contenidos. También pueden presentar abstracciones de forma gráfica. Exigen un procesamiento global de la información que contienen, y pueden producir un impacto emotivo que genere sentimientos y actitudes", Chou, O. (2008).

Se respaldaron en soporte digital los actos, las narraciones de cuentos y la música tradicional. En su creación intervinieron los participantes. Se utilizaron recursos módicos, música acorde con las motivaciones e intereses de estas edades. Este proyecto tuvo su génesis en un grupo de jóvenes amantes del senderismo y la Educación ambiental, transpolar sus gustos a los de los adultos fue un reto. "La música además de ser un recurso artístico, puede ser utilizada como herramienta pedagógica que favorece el desarrollo intelectual, motriz y de lenguaje, a través del fortalecimiento de dispositivos básicos del aprendizaje como la memoria, la atención, la percepción y la motivación” Díaz, M. L., Morales, R., \& Díaz, W. (2014).

La instrumentación de los medios audiovisuales en la formación y desarrollo de sentimientos de amor y cuidado hacia los recursos que brinda la naturaleza, constituyen una prioridad para la educación y autoeducación de la personalidad y de los intereses de la sociedad, lo cual es imprescindible. Se estableció todo un proyecto de turismo ecológico abarca una gran variedad de objetivos. Para constatarlo se requiere una transferibilidad de esta investigación en poblaciones similares, para:

- Fomentar lo que sería la promoción y atractivo turístico de un lugar.

- Aumentar y dar a conocer la oferta que existe para el turista.

- Captar eventos de cierta relevancia para la zona.

- Incentivar la implicación de todos los profesionales relacionados con el turismo para impulsarlo y atraer a mayor número de visitantes. 
RESULTADOS OBTENIDOS DE LA EDUCACIÓN AMBIENTAL A TRAVÉS DEL TURISMO ECOLÓGICO EN ADULTOS MAYORES EN LA LOCALIDAD DE FOMENTO CON EL USO MATERIAL AUDIOVISUAL.

Se pudo constatar que el turismo ecológico en esta población redujo en un porcentaje considerable los niveles de estrés generados en los adultos mayores. La recreación sana, así como el empleo del tiempo libre en técnicas de agricultura sostenible, lo facilitó. Es importante participar en este tipo de actividades porque "promueve la participación, debido a que las personas se relajan, aprenden cosas nuevas, comparten con otros, algunas actividades ejercen un efecto terapéutico que contribuye a la mantención de la salud." (Arévalo \& Yañez, 2016)

Las manifestaciones de tristeza y apatía, ante la realización de las tareas cotidianas, disminuyeron. Los adultos mayores realizaban su labor en la institución y en el hogar de una forma amena y con un nuevo sentido para sus vidas. Las enfermedades crónicas se mantuvieron estables, se disminuyó el riesgo por accidentes y la realización de ejercicios físicos moderados permitió desarrollar la elasticidad.

La percepción de los adultos mayores, que consideraban que el turismo no era apropiado para su edad, cambió al percatarse que la Educación Ambiental puede adaptarse a las discapacidades y potencialidades de estas edades. No cambiaron sus modos de alimentación, pero reconocieron la importancia de la ingesta de frutas, vegetales y la incorporaron a la rutina alimentaria.

Los adultos mayores de la muestra que presentaban problemas con el peso, pudieron evidenciar una tendencia paulatina a equilibrar el peso ideal, se sintieron más fuertes y vigorosos con el empleo de ejercicios físicos.

Los adultos mayores consideraban expresar su sexualidad como algo vergonzoso y con los grupos focales de discusión se evidenció cómo se manifestaba su vida sexual pasada, presente y futura. "la sexualidad, como el envejecimiento, son construcciones culturales y sociales, donde para su análisis se debe considerar una perspectiva de género y de derechos." Pedraza, T. (2014).

El turismo ecológico permitió la socialización de los adultos mayores que no presentan vínculo laboral y un acercamiento a sus familiares. "A través de un plan de acción ambiental a corto, mediano y largo plazo" (Hernández, Y. (2012).

Se desarrolló la motricidad, con las caminatas al aire libre. La terapia ocupacional con plantas deviene su utilidad de la base del ser humano como parte de lo natural; requiere que la persona se desempeñe dentro de un ambiente abierto y que se vincule con otro ser vivo en un contexto que entrega diversos estímulos sensoriales naturales, texturas, colores, olores, sonidos e incluso, el sabor requiere flexibilidad para asumir 
diversos roles, planificar y desplegar habilidades motoras. Es además una experiencia creativa.

\section{CONCLUSIONES}

En el análisis de los antecedentes y las investigaciones, se apreció cómo el turismo ecológico aún atraviesa por limitaciones y desafíos no resueltos, a tono con las transformaciones y perspectivas de la Educación ambiental y el adulto mayor.

El estudio realizado reveló que el turismo ecológico en el adulto mayor desarrolla la motricidad, reduce los niveles de estrés, disminuye la tristeza y apatía, estimula el pensamiento, estabiliza el peso corporal, permite manifestaciones de la sexualidad, la socialización, y desarrolla la motórica fina, estabiliza las enfermedades crónicas con la utilización de ejercicios, lo que repercute en mayor calidad de vida para el adulto mayor.

Los resultados de los estudios, realizados en el campo, en la localidad de Fomento, así como las acciones de reflexión colectivas, permitieron la construcción de una Estrategia Educativa de turismo ecológico que transforma la calidad de vida de los adultos mayores.

\section{COLABORADORES}

Dayana Gody Morffi Facultad de Economía. Universidad Central "Marta Abreu" de Las Villas, Villa Clara, Cuba

Dayana de la Caridad Hernández Heredia. Facultad de Economía. Universidad Central "Marta Abreu” de Las Villas, Villa Clara, Cuba. 


\section{REFERENCIAS}

Aguilar- Navarroa, S., \& Ávila-Funesa, J. A. (2007). La depresión: particularidades clínicas y consecuencias en el adulto mayor. Gacéta Médica de México, 143(2). Recuperado de http://www.medigraphic.com/cgi-bin/ new/resumen.cgi? IDARTICULO $=15149$

Arévalo, M., \& Yañez, J. (2016). Factores contextuales que influyen en la participación comunitaria en actividades de ocio y tiempo libre en personas en situación de discapacidad física. Revista chilena de terapia ocupacional, 16(2), 163-173 doi:10.5354/0719-5346.2016.44760

Castellanos, B. (1998). Investigación educativa, nuevos escenarios, nuevos actores, nuevas estrategias. Instituto Superior Pedagógico Enrique José Varona. Facultad de Ciencias de la Educación Centro de Estudios Educacionales.

Chou, O. (2008). Medios de enseñanza y tecnologías de la información. Cuba: Editorial Pueblo y Educación.

Coca, N., Martínez, P., \& Álvarez, M. (2016). Fábulas sobre agricultura sostenible para escolares con discapacidad intelectual. Revista Espacio $I+D$ Innovación más Desarrollo, Vol. 6 N²0, DOI http://dx.doi.org/10.31644/ IMASD.20.2019.a01

Cuba, (2015). Oficina Nacional de Estadísticas Cuba. Población. Recuperado de http:// www.one.cu.

Díaz, M. L., Morales, R., \& Díaz, W. (2014). La música como recurso pedagógico en la edad preescolar. Revista infancia imágenes. 13(1) doi:https:// doi.org/10.14483/16579089.5455

Fomento (2017) Informa de Dispensarización de la población por zona y consultorio médico. Material no publicado.

GalbánI, P. A., Soberats, F. J. S., \& Díaz-Canel, A. M. (2006). Envejecimiento poblacional y fragilidad en el adulto mayor. Revista Cubana de Salud Pública. Recuperado de http://www.scielo.org.pe/pdf/rspmi/v17n2/ a02v17n2

Hernández, Y. (2012). Acciones para propiciar una educación ambiental en el Consejo Popular "Simón Bolívar" del municipio Yaguajay (Tesis de pregrado) Universidad Central "Marta Abreu" de las Villas.

González, R. A., Yll, M., \& Curiel, L. D. (2003). Metodología de la Investigación Científica para las Ciencias Técnicas 1era. Parte: Diseño teórico y formulación del proyecto de investigación. Recuperado de https://www.academia.edu/13810811/Metodolog\%C3\%ADa_ de_la_Investigaci\%C3\%B3n_Cient\%C3\%ADfica_para_las_

Ciencias_T\%C3\%A9cnicas._Tomo_III

Kemmis. (1988). Educational research, methodology and measurement, an international handbook. Exeter Wheaton \& Co. Recuperado de https:// 
s3-euw1-ap-pe-ws4-capi2-distribution-p.s3-eu-est1.amazonaws.com/bo oks/9781135389291/9781135389291_previewPDF

Kulkarni, T., Karajgi, A., \& Pandit, U. (2018). Comparison between virtual reality training using $\mathrm{x}$-box 360 kinect and conventional physiotherapy on trunk, postural control and quality of life in chronic stroke survivals. International Journal of Current Advanced Research, 7(4). doi: http:// dx.doi.org/10.24327/ijcar.2018.11577.2006

Llanes, R. (2012). Glosario de filosofía, economía y política. Cuba: Editorial Ciencias Médicas.

Mercedes, A., Sánchez, L., Somonte, R., \& Cabrera, A. M. (2014). Envejecimiento poblacional: una mirada desde los programas y políticas públicas de América Latina, Europa y Asia. Revista Novedades en Población, 10(19). Recuperado de http://scielo.sld.cu/scielo.php?script=sci_arttext \&pid=S1817-40782014000100002

Nico, M. R. (2016). Desórdenes de la modulación sensorial y dibujo de la figura humana. Revista chilena de terapia ocupacional, 16(1). doi:10.5354/0719- 5346.2018.48088

Pedraza, T. (2014). Vejez y sexualidad: reflexiones para la práctica de terapia ocupacional. Revista chilena de terapia ocupacional, 14(2). Recuperado de https://revistaterapiaocupacional.uchile.cl/index.php/RTO/article/ view/35726

Pérez, A. (2010). Propuesta de acción para proyectar la Educación ambiental en el Consejo Popular "Abel Santa María" desde el desarrollo comunitario. (Tesis de pregrado) Universidad Central "Marta Abreu" de las Villas.

Ricardo, D. (2006). Ecología, ecologismo y Medio Ambiente. Revista Luna Azul, 25/08/2006. Recuperado de http://lunazul.ucaldas.edu.co/ index2.php?option=com_content\&task=view\&id=279\&I

Rodríguez, G., Gil, J., \& García, E. (1996). Metodología de la investigación cualitativa. Recuperado de https://www.researchgate.net/publication/44376485_Metodologia_de_la_investigacion_cualitativa_Gregorio_ Rodriguez_Gomez_Javier_Gil_Flores_Eduardo_Garcia_Jimenez

Ruiz, A. (1999a). Invest- educación Introducción a la investigación en la educación. Cuba: Editorial Pueblo y Educación.

Ruiz, A. (1999b). La triangulación. Editorial Chapecó. Metodología de la investigación. Educativa. Brazil.

Ruiz, A. (2000). INVEST- EDUCACIÓN Introducción a la investigación en la educación. Cuba: Editorial Pueblo y Educación.

Sampier, R. H. (2006). Metodología de la investigación. México: Hill Interamerica Editores.

Silva, C. R., Cardinalli, I., Silvestrini, M. S., Farias, A. Z., Prado, A. C. d. S. A., Ambrosio, L., Paula, B. M. d. (2017). La terapia ocupacional y la cultura: miradas a la transformación socialcerebral; una re- 
visión sistemática. Revista chilena de terapia ocupacional, 105-113 doi:10.5354/0719-5346.2017.46383

Silva, R. (2007). Métodos cualitativos de investigación. Recuperado de http://www.sepiensa.cl/edicion

Taylor, S. J., \& Bogdan, R. (1987). Introducción a los métodos cualitativos de investigación. La búsqueda de significados. In PAIDos. Recuperado de http://wwwTaylor-Bogdan_observacion_part.pdf_AWSAcces sKeyId=AKIAIWOWYYGZ2Y53UL3A\&Expires=1540393526\&Sig nature $=7$ VoXLtFbXbVndjvcFs58eF7Ezig\%3D\&response-contentdisposition=inline\%3Bfilename\%3DTaylor_Bogdan_observacion_part

Valle, A. D. (2007). Metamodelos de la investigación pedagógica. Cuba: Editorial Pueblo y Educación.

Vera, M. (2007). Significado de la calidad de vida del adulto mayor para sí mismo y para su familia. Facultad de Medicina, 68(3). Recuperado de http://www. scielo.org.pe/scielo.php?pid=S1025-55832007000300012\&script=sci_arttext

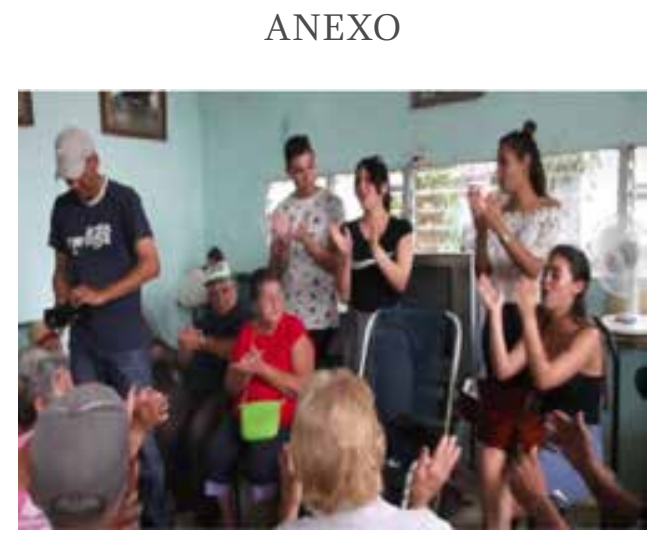

Fotografía 1. Actos culturales

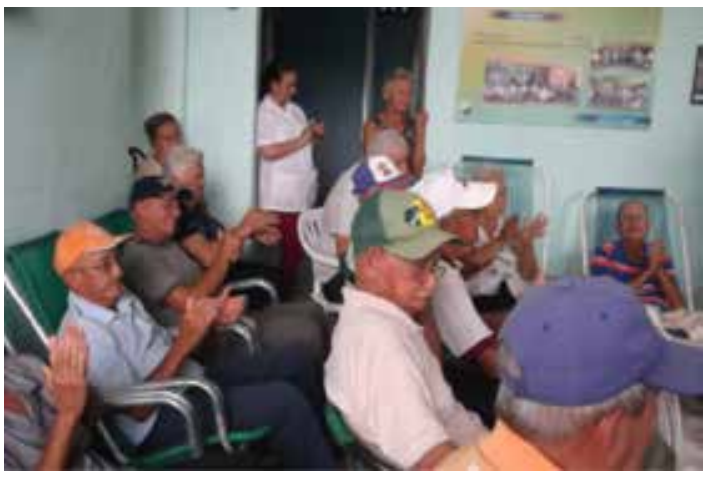

Fotografía 2. Construcción de cuentos de la localidad 